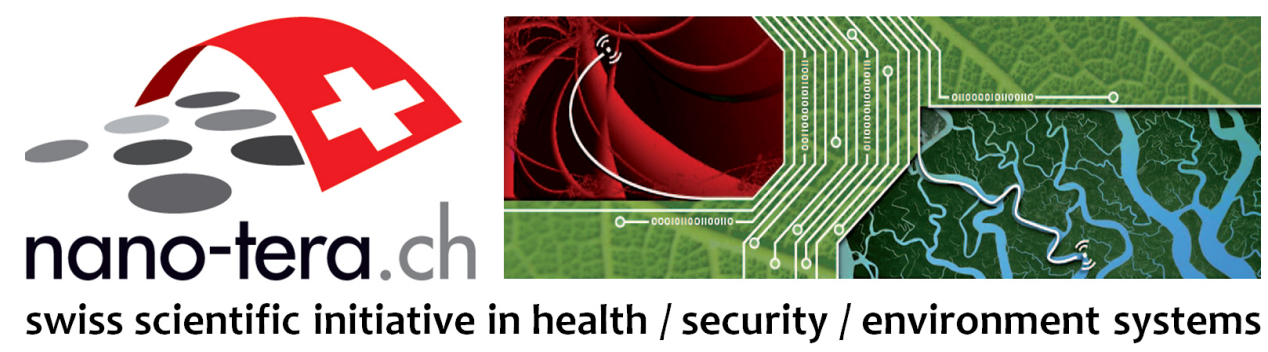

P. Mayor, P. Bradley, G. De Micheli, Solid-State Circuits Magazine IEEE 2, no.3, 87-92 (2010)

\title{
Nano-Tera.ch: Engineering Complex Systems for Health, Security, and the Environment
}

The Nano-Tera.ch initiative is a collaborative engineering program that fosters research and crossbreeding of hardware and software technologies in the areas of implantable, wearable, and ambient systems. The scientific outcome is expected to better the quality of health, security, and environmental systems; to foster a vision of engineering with social objectives; and to promote related educational programs.

This broad Swiss engineering program is currently funded by the Swiss government with a CHF60 million ( US\$54 million) cash grant for the period 2008-2011 and also by CHF60 million of matching support from participating and third-party institutions. Nano-Tera.ch is a consortium whose partners are the two Swiss Federal Institutes of Technology (EPFL and ETHZ), the Swiss Center for Electronics and Microtechnology (CSEM), as well as the Universities of Basel, Geneva, Lugano, and Neuchâtel. The consortium provides a means to fund research to all Swiss universities and nonprofit research centers. The Swiss National Science Foundation (SNSF) plays a key role in the evaluation of the large-scale projects.

The mission of this program can be summarized as the research, design, and engineering of complex (terascale) systems using micro/ nanotechnologies to monitor and connect humans and/or the environment through distributed and networked systems and services. The program aims at advancing engineering research in nanoscale materials/ devices design and in their exploitation in a variety of systems, requiring heterogeneous integration and coordinated control of diverse micro/nanoscale components. Embodiments of such systems can be found in lightweight, mobile, and personalized products embedded in the environment and on/in the body. These products will enable us, for example, to detect in real time different health risks and conditions through integrated bio-probing, to reveal security risks through smart buildings and environments, to save energy through ambient sensing, and to detect and monitor environmental hazards, such as floods and avalanches. The outstanding novelty and power of these systems stem from their connectedness and the integration of heterogeneous components and technologies. Thus, the thrust of the program is concentrated on the formation of collaborative, interdisciplinary, and interinstitutional teams of scientists.

\section{The Application Areas...}

As diverse as tomorrow's society constituent groups may be, they will share the common goal of a safer and healthier lifestyle. Electronic systems and services will play a major role in supporting higher levels of working effectiveness, better communication, and personal interaction. Wearable embedded systems will provide the gateway between humans and information systems 


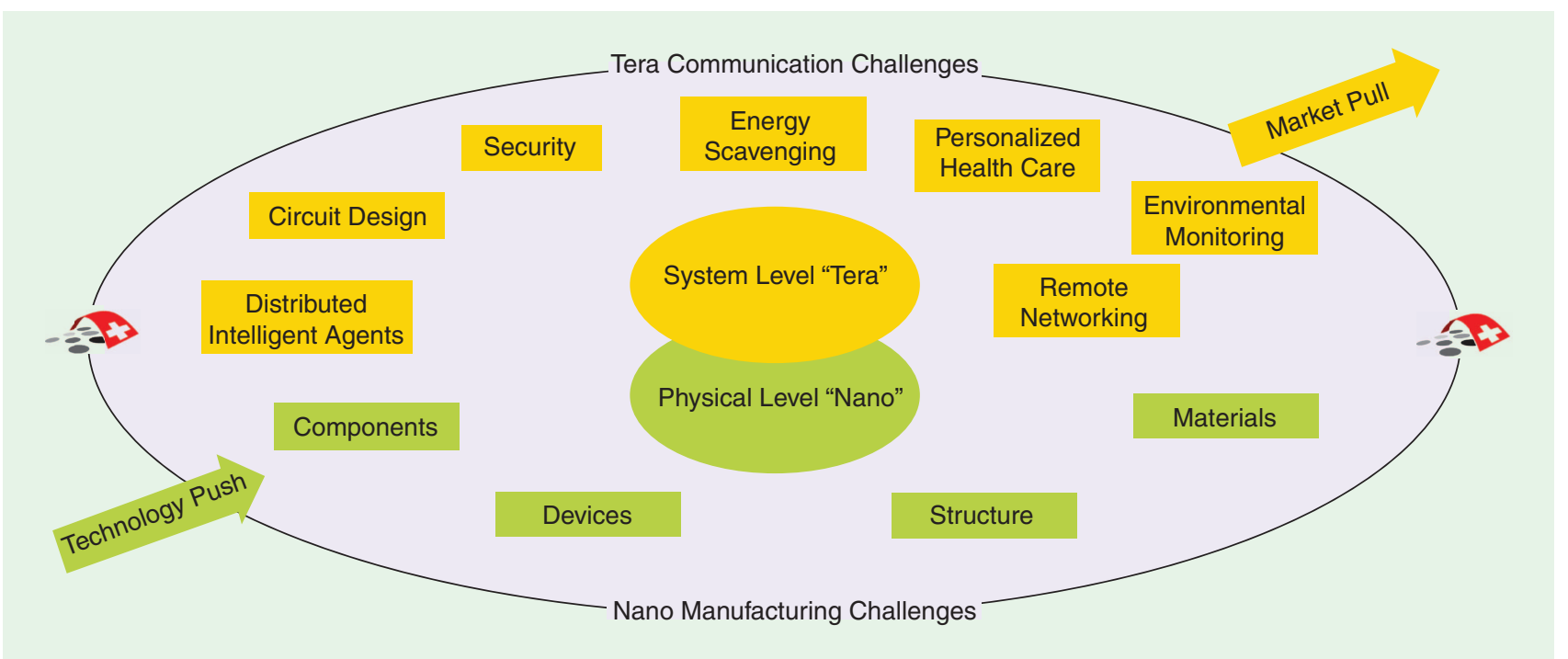

The Nano-Tera.ch challenge is to bridge advancement in technology with the requirement of increasingly sophisticated systems and services addressing societal needs. Thus Nano-Tera.ch fosters groundbreaking engineering research objectives through the convergence of scientists and technologists with different backgrounds.

and will be characterized by long periods of autonomy, complex functionality, and substantial computational power. Within Nano-Tera. ch, specific application targets of wearable systems are in the fields of monitoring of professional and recreational sportsmen, diagnosis and/or treatment of patients, and, more generally, enhancement of security and safety of individuals. Thus, the wearable application area has a significant overlap with the domain of electronic and personalized health management through the use of implanted devices, smart clothing, and intelligent nutrition/drug monitoring and delivery systems.

Ambient systems constitute another key application area of Nano-Tera. ch, encompassing large-scale heterogeneous systems used to sense, network, inform, actuate, and/or interact with the physical environment, devices, or humans. These systems are at the heart of nextgeneration information technology, which will no longer be limited to dedicated infrastructures (e.g., the Internet) but will consist of highly distributed, networked, heterogeneous, and largely self-organizing devices and will be embedded into the environment. Ambient systems research addresses environmental monitoring, smart buildings, workplaces and transportation systems, and virtualworld applications. The combination of ambient and wearable systems provides us with platforms to enhance human security, for example, by monitoring elderly or disabled persons in their homes.

\section{... and Their Enabling Technologies}

The Nano-Tera.ch program is articulated into projects. We describe some of them as examples of its technology areas of interest. A full list of major projects is reported in Table 1.

\section{Micro/Nano-Electronics}

With complementary metal-oxidesemiconductor (CMOS) technology scaling becoming more difficult and expensive, micro/nanoelectronic research within Nano-Tera.ch is characterized by the search for new applications of semiconductor technologies with extended functionalities and combined technology-system innovation. In essence, Nano-Tera.ch has been focusing on the "More Than Moore" avenue, with the realization of sensors and micro/nano electro-mechanical systems (M/NEMS) and their integration with micro/nano-electronic circuits, as well the design of new devices in new technologies (such as silicon nanowires and carbon nanotubes) that can leapfrog extremely downscaled CMOS. Besides providing platforms for aggressively downscaled electronic technologies, research is also addressing threedimensional (3-D) integration as a means of realizing heterogeneous integrated systems.

The PlaCiTUS project is motivated by the observation that the aggressive downscaling of CMOS technology to the nanoscale device size and terascale system complexity has been obtained at the cost of severe degradation of certain quality metrics, such as the increase of parameter variability, strong degradation of device matching, and increase in leakage currents. The profound changes in the device structure that are required to mitigate or even circumvent all these degradations will obviously have a significant impact on the way circuits, particularly analog and RF circuits, have to be designed. This project investigates the challenges in mixed signal platforms from both device and systems perspectives. Ultimately, the nano-CMOS design platform will 
provide us with the different devices required for health, security, and environment applications with much smaller size and longer autonomy.

Interestingly, 3-D integrated circuits are attractive solutions for overcoming present barriers encountered in interconnect scaling. The CMOSAIC project is a genuine opportunity to contribute to the realization of arguably the most complicated system that mankind has ever assembled: a 3-D stack of silicon chips with a functionality per unit volume that nearly parallels that of the human brain. By compressing almost $10^{12}$ nanometersized functional units into a $1-\mathrm{cm}^{3}$ volume with a $10-100$ fold higher connectivity

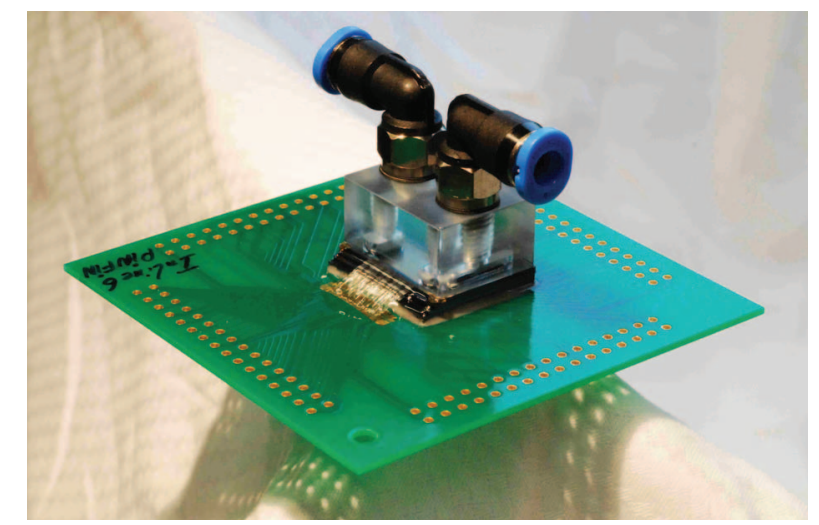

Three-dimensional stack with inter-tier cooling and wire bonding, used in the CMOSAIC project. where the main challenge is to remove the heat produced by multiple stacked dies with each layer dissipating $100-150 \mathrm{~W} / \mathrm{cm}^{2}$. Therefore, state-of-the-art microscale singlephase liquid and two-phase cooling systems are being developed,

two-phase environmentally friendly refrigerants and novel nanocoated, nonwetting surfaces. In layman's terms, computation will be enabled by the heat extraction provided by boiling coolant in microchannels, thus providing a formidable mix of technologies which are addressed by a multidisciplinary team of scientists.

\section{Sensors}

The general objectives of Nano-Tera.ch to create systems for environmental and biomedical applications require new sensors and their seamless integration with micro/nanoelectronic technology. Until now, most sensors were usually developed as stand-alone devices, with the notable exception of imaging devices (as seen in

using specifically designed microchannel arrangements with channel sizes as small as $50 \mu \mathrm{m}$. The employed cooling means include digital cameras and mobile phones) and automotive applications. Most other sensors, such as chemical gas, liquid, or glucose-level sensors, have for such high-performance systems

\section{LARGE-SCALE COLLABORATIVE INTERDISCIPLINARY NANO-TERA.Ch RESEARCH PROJECTS IN PROGRESS.}

More details on these projects, as well as on small-scale and educational activities, can be found at www.Nano-tera.Ch.

CabTuRes: Low-power nano-sensor/electronics building blocks based on tunable CNT electro-mechanical resonators

C. Hierold, ETHZ

CMOSAIC: Three-dimensional stacked architectures with interlayer cooling

GreenPower: Connecting renewable energy to green mobility using hydrogen as energy carrier

i-IronIC: Implantable/wearable system for online monitoring of human metabolic conditions

IrSens: Integrated sensing platform for gases and liquids in the near and midinfrared range

ISyPeM: Intelligent integrated systems for personalized medicine

LiveSense: Cell-based sensing microsystem

MIXSEL: Vertical integration of ultrafast semiconductor lasers for wafer-scale mass production

NanowireSensor: Integrateable silicon nanowire sensor platform

Nexray: Network of integrated miniaturized X-ray systems operating in complex environments

NutriChip: A technological platform for nutrition analysis to promote healthy food

OpenSense: Open sensor networks for air quality monitoring

PATLiSci: Probe array technology for life science applications

PlaCiTUS: Platform circuit technology underlying heterogeneous nano- and terasystems

QCrypt: Secure high-speed communication based on quantum key distribution

SelfSys: Fluidic-mediated self-assembly for hybrid functional micro/nanosystems

SImOS: Smart implants for orthopaedics surgery

TecInTex: Technology integration into textiles: empowering health

$X$-Sense: Monitoring alpine mass movements at multiple scales
J. Thome, EPFL

J.-A. Månson, EPFL

G. De Micheli, EPFL

J. Faist, ETHZ

C. Guiducci, EPFL

P. Renaud, EPFL

U. Keller, ETHZ

C. Schönenberger, Uni Basel

A. Dommann, CSEM

M. Gijs, EPFL

K. Aberer, EPFL

H. Heinzelmann, CSEM

Q. Huang, ETHZ

N. Gisin, Uni Geneva

J. Brugger, EPFL

P. Ryser, EPFL

G. Tröster, ETHZ

L. Thiele, ETHZ 
been developed mainly for laboratory analytical instrumentation, with very few integrated applications. Thus, important new challenges for sensors and their integration include ultralow powered cantilever or nanotubes arrays, single photon detectors, celland microfluidics-based chips, and biocompatible coatings.

An example of a project in this category is i-IronIC, which seeks to develop integrated smart biosensors for online metabolism monitoring that significantly improves the quality and reliability of human measurements while at the same time reducing analysis time and cost. The new system will investigate many different metabolic compounds of interest for cardiovascular and inflammatory diseases as well as for personalized nutrition, such as lactate, cholesterol, and ATP. To pursue this aim, an innovative technology is being developed to integrate sensing, circuits, and software in three devices: a fully implantable sensor array for data acquisition, a wearable station for wireless powering and signal processing, and a remote station for data collection and storage. This advanced and reliable diagnostics implantable system will be useful for the individualization of therapies and for disease prevention and nutrition in patients, athletes, and the elderly.

The project IrSens responds to the increasing demand for sensitive and portable detectors for trace components in gases and liquids (e.g., for atmospheric pollutants or drug detection) by building a versatile platform based on optical spectroscopy in the near and midinfrared range. Indeed, techniques based on optical absorption allow us to probe the vibrational frequencies of the targeted molecules-most of which are located in the near and midinfrared range-and to obtain an unambiguous signature of the investigated gas or liquid. The objective is to create a new sensor element and platform with vastly improved performance and lower cost.

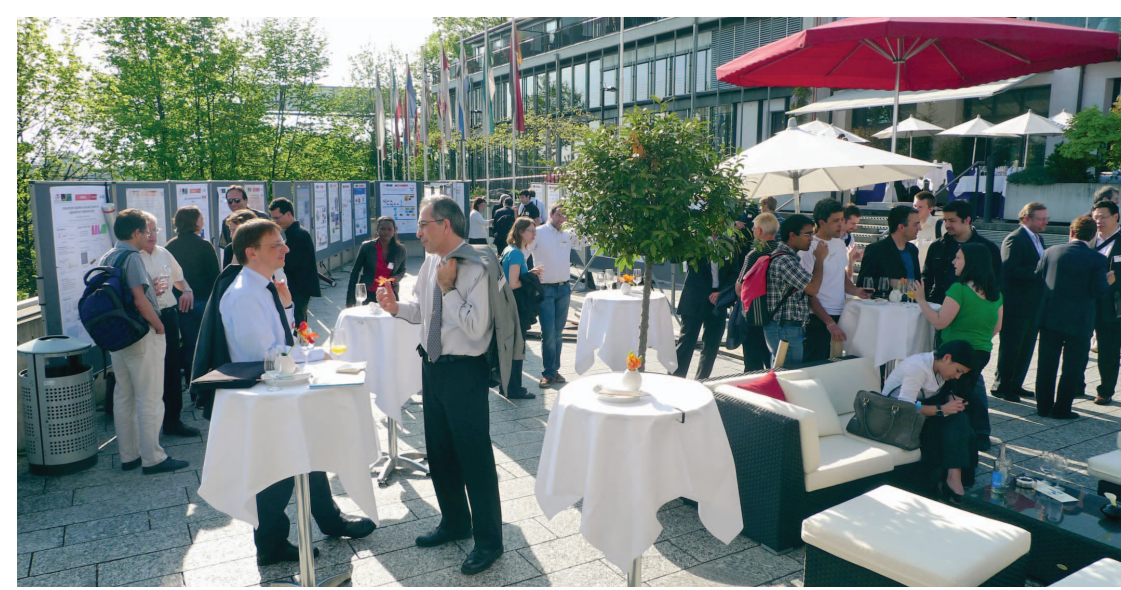

Nano-Tera.ch held its first annual meeting on 29 April 2010, gathering over 200 researchers and industrial partners involved in its multifarious projects.

\section{MEMS/NEMS}

Micro/nano electromechanical systems provide interface functions (sensors and actuators) between micro- or nanoelectronics and the environment or living beings. There is therefore a strong relation between sensors and M/NEMS. Today's prominent examples are provided by macro-molecular carbon nanotubes, which have already been used for displacement, force, pressure, and gas sensing. Moreover, M/NEMS are enabling devices all kinds of terascale systems and other functions, such as energy sources. Indeed, energy harvesters based on the extraction of vibrational energy have been shown to be successful in various domains. M/NEMS will also become part of micro- and nano-electronic systems (systems-in-package) providing functions like resonators, filters, switches, and RF components.

Carbon nanotubes (CNTs) are a perfect example of how nanomaterials offer new features that had been unachievable: their outstanding structural, mechanical, and electronic properties have immediately resulted in numerous device demonstrators from transistors, to physical and chemical sensors, and actuators. Due to their small mass and high stiffness, doubly clamped CNTs can exhibit very high resonant frequencies. As recently demonstrated or predicted, these carbon nanotubes can reach the multi-gigahertz range, can be tuned via straining over a wide range of frequency, offer an unprecedented sensitivity to strain or mass loading, and exhibit high quality factors. In this context, the project CabTuRes seeks to demonstrate concepts and devices for ultra-low power, highly miniaturized functional blocks for sensing and electronics. Indeed, carbon nanotube resonators offer a wide range of electronics applications that allow them to be used as tunable voltage controlled oscillators, clocks, or nano electromechanical filters and detectors. Moreover, the high sensitivity of the resonant frequency to tiny mass variations makes it possible to use the resonators to measure gas molecule densities or weigh nano bodies such as proteins and viruses.

Techniques based on micromechanical force sensors (microcantilevers) are also of increasing importance for applications in biological sciences. Their nanomechanical properties enable us to use them as highly sensitive probes for the detection of molecular species adsorbed to them. The project PATLiSci seeks to enhance probe-array techniques for 
life-science applications, notably in the context of cancer research. Indeed, it has been shown recently that the stiffness of cancer cells and their adhesion forces affect the way they spread in the body. The measurement of nanomechanical properties of cells as well as cell-to-cell interactions as a function of milieu parameters is thus of particular interest in cancer research. The project is expected to advance personalized medical diagnostics, with a direct impact on pharmacological research and on cell-based drug screening.

\section{Software, Information, and Communication}

Terascale distributed embedded systems involve huge sets of nodes performing information acquisition, processing, and communication, often in a self-organized manner, under the constraints of operating at high speed and with low power consumption. Algorithms and software play a major role in integrating the distributed components and guaranteeing the desired overall system behavior. Technologies for correct software design production, including the ability to formally prove its correctness, are key for sustaining terascale system operation and security. At the same time, information and communication technologies face tremendous challenges, as terascale distributed information processing systems generate unprecedented amounts of data, which needs to be processed, stored, and efficiently transmitted over large-scale highly heterogeneous wireless networks. Terascale networks need to cope with large scales of small and unreliable components and provide safe and reliable results.

The project OpenSense addresses key research challenges in the domain of information and communication systems related to community-based sensing using wireless sensor network technology in the context of air pollution monitoring. Solutions to these problems typically affect all layers of an information and communication system architecture, with interdependencies and synergies among the different layers. OpenSense will result in an open technology that allows for the integration of diverse sensors, including mobile sensors, into a single environmental model. The information processing techniques will provide important insights into other application domains dealing with monitoring complex events. The ultimate goal is to provide an open and extensible platform for monitoring air quality in real time, for better understanding environmental phenomena and their effects on individuals and communities.

Monitoring alpine mass movements has attained crucial importance, as climate change results in destructive geological processes influencing the stability of slopes and inducing landslides. The project X-Sense addresses these issues by developing a monitoring and warning system for the spatial and temporal detection of newly forming hazards and by extending the quantitative understanding of these changing natural systems and our predictive capabilities. The project will develop dependable wireless sensing technology as a new scientific instrument for environmental sensing under extreme conditions and unattended operation as required for long-term deployment. This technology integrates various sensing dimensions to extend the spatial scope from local (microscopic) measurements to large-scale information derived from satellite radar remote sensing and to fuse the resulting information to achieve an unparalleled degree of precision in space, time, and accuracy. Key research involves advanced differential global positioning system sensing for high-precision movement detection and the development of sensor fusion algorithms combining different classes of sensors with high spatial granularity and satellite-scale $\mathrm{X}$-ray images.

\section{Looking Toward the Future}

Involving more than 500 scientists and researchers within 28 institutions in Switzerland, Nano-Tera.ch has generated a large momentum in the transfer of scientific discoveries to the engineering domain, for example, the use of new materials and devices for sensing and computation. Nano-Tera.ch looks forward to creating networks of devices for gathering collective information about phenomena that are particularly important in the domains of health, security, and environment. Having been active for over two years, the program hosted a the recent annual meeting in Bern that attracted a large number of attendees from university and research centers.

As Nano-Tera.ch plans its extension for 2012-2016, an application area is emerging as central to interdisciplinary research: energy: The smart creation, distribution, and consumption of energy that is key in many aspects of our society- from the data centers to implanted electronics and to environmental wireless monitoring networks, it is crucial to acquire, process, and transfer data with optimal energy efficiency.

Beside mere scientific objectives and their related educational programs, we believe that the topics of research within Nano-Tera.ch have a strong societal and ethical dimension that will help attract students to engineering and broaden their perspectives.

\section{-Patrick Mayor \\ Peter Bradley, and Giovanni De Micheli}
About the Authors
Patrick Mayor is the scientific coor- dinator and reporter for Nano-Tera. ch. He is responsible for the com- munication and the dissemination of the research conducted within the program. He holds a Ph.D. degree in physics from the Swiss Federal 
Institute of Technology in Lausanne (EPFL). His research focused on the central concept of temperature in granular materials, introducing a method to probe these out-of-equilibrium systems that has become well known in the field. He was a post-doctoral fellow for two years at the University of Pennsylvania before joining the Nano-Tera.ch team.

Peter Bradley is the executive director of Nano-Tera.ch. His responsibilities include operational management, coordination with the various bodies of authority, steering industrial interest and partnership with close relationship at the industrial, diplo- matic and academic level. He is also taking strategic initiatives for setting up the development of a knowledgebased community around this unique engineering-focused national program. He founded AMT Intelligence, Geneva, in 2006. He received his Ph.D. degree in materials science and engineering with special honors from EPFL, Switzerland, in 1998.

Giovanni De Micheli is the chair and program leader of Nano-Tera.ch. He is also a professor and director of the Institute of Electrical Engineering and of the Integrated Systems Centre at EPFL, Switzerland. Previously, he was a professor of electrical engineering at Stanford University. His research interests include several aspects of design technologies for integrated circuits and systems, such as synthesis for emerging technologies, networks on chips and 3-D integration. He is also interested in heterogeneous platform design including electrical components and biosensors, as well as in data processing of biomedical information. He is the author of Synthesis and Optimization of Digital Circuits (McGrawHill, 1994), coauthor and/or coeditor of eight other books and of over 400 technical articles. 